\title{
Geomagnetic field variability: geomagnetic dynamo and helical distributed chaos
}

\author{
A. Bershadskii \\ ICAR, P.O. Box 31155, Jerusalem 91000, Israel \\ e-mail: bershads@gmail.com
}

\begin{abstract}
It is shown that helicity dynamics dominates the thermal convection-driven geomagnetic field variability. The notion of the distributed chaos has been used for this purpose. The large-scale circulation in the Earth's outer (metallic liquid) core is especially sensitive to the helicity dynamics and its chaotic reversals can be associated with the reversals of the mean helicity sign (the ergodic restoration of the reflectional symmetry in a long run). Results of numerical simulations, laboratory and atmospheric experiments, and observations of the crustal (the World Magnetic Anomaly Map) and paleomagnetic fields (the geomagnetic field dipole variability and reversals) have been used in order to support the theoretical considerations based on the adiabatic invariance of the second order moment of the helicity distribution.
\end{abstract}

\section{INRODUCTION}

The global geomagnetic field plays important role in sustaining life on the Earth providing a shield from the action of the solar wind [1], 2]. The modern technology, based on the electromagnetic processes, is also effectively shielded from the solar wind effects by the global geomagnetic field. However, the global geomagnetic field intensity is varying in time, and although the characteristic time scales of this variability are usually large (or very large) in comparing with the human lifetime, it seems to be important to understand its temporal dynamics. The complete polarity reversals and excursions are of especial interest (see, for instance, Ref. [3] and references therein).

It is believed that the global geomagnetic field is generated by a thermal convection-driven geomagnetic dynamo mechanism in the outer (liquid and electricallyconducting) core of the Earth [3]. Since the thermal convection is the main energy source for the geomagnetic dynamo we will first of all consider some relevant properties of the strong thermal convection.

It will be shown (Section II) that although helicity is not conserved in the thermal convection (even in the inviscid approximation) the second order moment of the helicity distribution (the Levich-Tsinober invariant of the Euler equation [4], [5]) is an inviscid invariant of the thermal convection at certain (rather general) conditions. Application of the Kolmogorov-like phenomenology using this adiabatic invariant results in the scaling power spectrum $E(k) \propto k^{-4 / 3}$ for the velocity fluctuations in the inertial range of scales (Section III). Usually the scaling inertial range is not large (if it appears at all) for the velocity fluctuations in the turbulent thermal convection and the distributed chaos approach is often providing a more adequate description of the processes (especially for the moderate values of the Rayleigh number). Corresponding to this adiabatic invariant power spectrum $E(k) \propto \exp -\left(k / k_{\beta}\right)^{1 / 3}$ will be obtained for the velocity fluctuations in the frames of the distributed chaos approach (Section IV).

A large-scale circulation (clockwise or anticlockwise global circular wind) can appear in the turbulent Rayleigh-Bénard convection at sufficiently large Rayleigh numbers (see, for instance, Refs, [6], 7] and references therein). Appearance of such global circulation in a cylindrical cell, for instance, violates the reflectional spatial symmetry. Natural attempts of the system to restore the reflecional symmetry in a long run result in the abrupt chaotic [8] reversals of the direction of the large-scale circulation. It is shown (Section V) that the large-scale circulation is especially sensitive to the helicity dynamics and the reversals of the direction of the circulation can be associated with corresponding reversals of the mean helicity's sign.

In Section VI analogous properties of the thermal convection-driven magneto-hydrodynamic (MHD) dynamo will be considered and compared with results of a numerical simulation relevant to the Earth's outer (liquid) core. In Section VII results of this consideration will be compared with the spatial (wavenumber) spectrum of the crustal magnetic filed computed using the World Magnetic Anomaly Map (WMAM [9]).

In Section VIII this consideration will be extended on the paleomagnetic dipole variability and compared with results on corresponding numerical simulations and composite power spectra of geomagnetic dipole moment variations measured for the total time interval 0-160 Myr.

\section{ADIABATIC INVARIANTS}

In the Boussinesq approximation thermal (buoyancy driven) convection is described by equations [10]

$$
\begin{gathered}
\frac{\partial \mathbf{u}}{\partial t}+(\mathbf{u} \cdot \nabla) \mathbf{u}=-\frac{\nabla p}{\rho_{0}}+\sigma g \theta \mathbf{e}_{z}+\nu \nabla^{2} \mathbf{u} \\
\frac{\partial \theta}{\partial t}+(\mathbf{u} \cdot \nabla) \theta=S \frac{\Delta}{H} e_{z} u_{z}+\kappa \nabla^{2} \theta,
\end{gathered}
$$




$$
\nabla \cdot \mathbf{u}=\mathbf{0}
$$

where $\theta$ is the temperature fluctuations (over the temperature profile), $\mathbf{u}$ is the velocity and $p$ is the pressure, $\mathbf{e}_{z}$ is a unit vector (along the gravity direction) and $g$ is the gravity acceleration, $H$ and $\Delta$ are the distance between the layers and the temperature difference between the layers, the mean density is denoted as $\rho_{0}$, whereas $\nu, \kappa$ and $\sigma$ are the viscosity, thermal diffusivity and thermal expansion coefficient. For the unstable stratification (Rayleigh-Bénard convection) $S=+1$ whereas for the stable stratification $S=-1$.

In the non-dissipative approximation $(\nu=\kappa=0)$ equations (1-3) have a generalized energy invariant

$$
\mathcal{E}=\int_{V}\left(\mathbf{u}^{2}-S \sigma g \frac{H}{\Delta} \theta^{2}\right) d \mathbf{r}
$$

where $V$ is the spatial domain's volume [10]).

At certain conditions the Eqs (1-3) have an additional inviscid invariant. For $\nu=0$ equation for mean helicity is

$$
\frac{d\langle h\rangle}{d t}=2 \sigma g e_{z}\left\langle\omega_{z} \theta\right\rangle
$$

(the helicity density $-h=\mathbf{u} \cdot \boldsymbol{\omega}$, the vorticity $-\boldsymbol{\omega}=\nabla \times \mathbf{u}$, $\langle\ldots\rangle$ - average over the spatial volume $V$ ), hence the helicity is not an inviscid invariant of the thermal convection. Let us recall that the mean helicity together with the mean energy are the fundamental invariants for the Euler equations [5]. The Euler equations have an additional invariant - the second order moment of the helicity distribution (the Levich-Tsinober invariant [4], [5]). In the thermal convection the main contribution to the correlation $\left\langle\omega_{z} \theta\right\rangle$ (from the Eq. (5)) comes from the large-scale motion (mainly from the coherent structures) and it can be rather considerable, but the correlation between $\omega_{z}$ and $\theta$ is quickly diminished with decreasing spatial scales due to the turbulent effects. In order to take into account this phenomenon let us consider the second order moment of the helicity distribution. To define this moment let us divide the spatial domain of motion into the cells (with volumes $V_{j}$ and the boundary conditions $\boldsymbol{\omega} \cdot \mathbf{n}=0$ on the surfaces of the cells) moving with the fluid [5]. The second order moment can be then defined as [5]

$$
I=\lim _{V \rightarrow \infty} \frac{1}{V} \sum_{j} H_{j}^{2}
$$

where

$$
H_{j}=\int_{V_{j}} h(\mathbf{r}, t) d \mathbf{r} .
$$

Due to above mentioned phenomenon the $H_{j}$ are inviscid quasi-invariants for the cells with small enough spatial scales. For sufficiently developed turbulence such cells provide the main contribution to the sum Eq. (6) (cf. Ref. [11]) and, as a consequence, the total sum in the Eq. (6) is also a quasi-invariant at $\nu=0$.

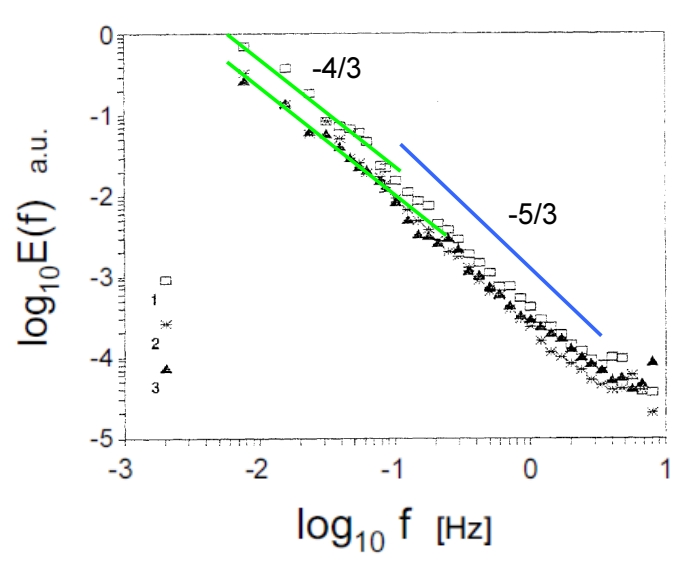

FIG. 1: Power spectrum of the longitudinal velocity fluctuations in the atmospheric near free convection.

\section{SCALING SPECTRA OF THE VELOCITY FLUCTUATIONS}

In the frames of the Kolmogorov-Obukhov phenomenology [12] scaling spectra of the velocity fluctuations in the inertial range of scales can be estimated as

$$
E(k) \propto \varepsilon^{2 / 3} k^{-5 / 3},
$$

where

$$
\varepsilon=\left|\frac{d\left\langle\mathbf{u}^{2}-S \sigma g \frac{d}{\Delta} \theta^{2}\right\rangle}{d t}\right|
$$

and the generalized energy Eq. (4) is considered as an adiabatic invariant in the inertial range of scales.

In order to apply this approach using the second order moment of the distribution of the helicity density - $I$ (Eq. (6)), as an adiabatic invariant in the inertial range of scales we should take into account that (unlike the energy, which is a quadratic invariant) the second order moment $I$ is a quartic invariant (cf. Eq. (6)). Therefore, we should use $\varepsilon_{I}=\left|d I^{1 / 2} / d t\right|$ instead of $\varepsilon$, and we obtain from the dimensional considerations

$$
E(k) \propto \varepsilon_{I}^{2 / 3} k^{-4 / 3}
$$

Results of atmospheric measurements of the longitudinal velocity fluctuations in the near free convection (Rayleigh-Bénard convection, i.e. the unstable stratification conditions) were reported in the Ref. [13]. The measurements were made by a probe at the height $\sim$ $12 \mathrm{~m}$ above sea surface. The surface was aerodynamically smooth and the weather was calm. The results for three data sets with the wind gusts comparable to the r.m.s. horizontal velocity fluctuations (near free convection) are shown in the log-log scales in Figure 1 (the spectral data for the Fig. 1 have been taken from Fig. 1a of the Ref. [13]). 


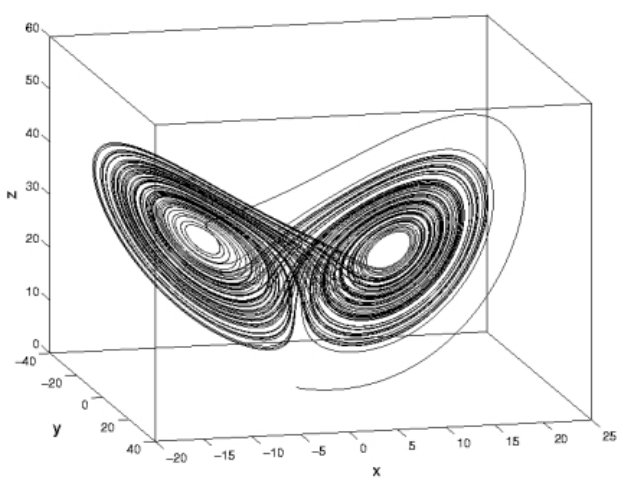

FIG. 2: A typical example of Lorenz chaotic attractor.

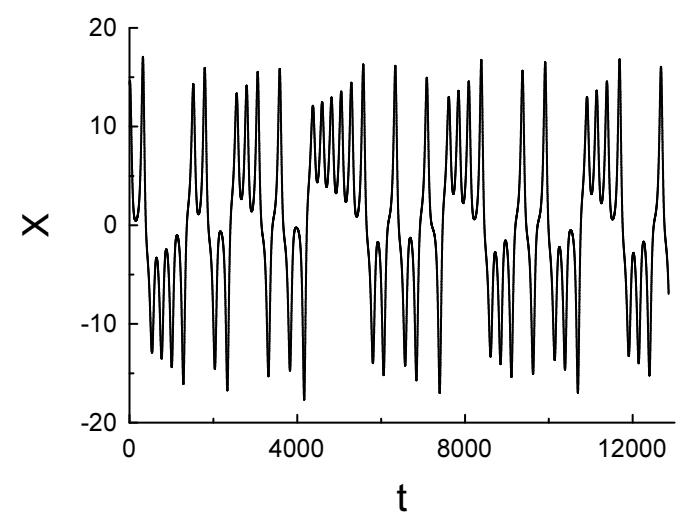

FIG. 3: A sample of the $X(t)$ time series for the Lorenz system.

In these measurement the time signals were taken by probes at a fixed spatial location and reflect characteristics of the spatial structures moving past the probes. As a consequence the observed frequency spectra actually reflect the wavenumber spectra (so-called Taylor hypothesis [12]) with the replacement $f=U_{0} k / 2 \pi$, where $U_{0}$ is usually a mean velocity. In the case of the thermal convection the mean velocity can be replaced by a characteristic velocity of advection by the energy-containing eddies past the probe [14], 15]. Therefore, the solid straight lines are drawn in the Fig. 1 to indicate the scaling spectra: Eq. (8) for the small-scale part of the inertial range and Eq. (10) for the large-scale part of the inertial range.

\section{DISTRIBUTED CHAOS}

Let us start from a simple (Lorenz-) model of the thermal convection considering only the first modes in

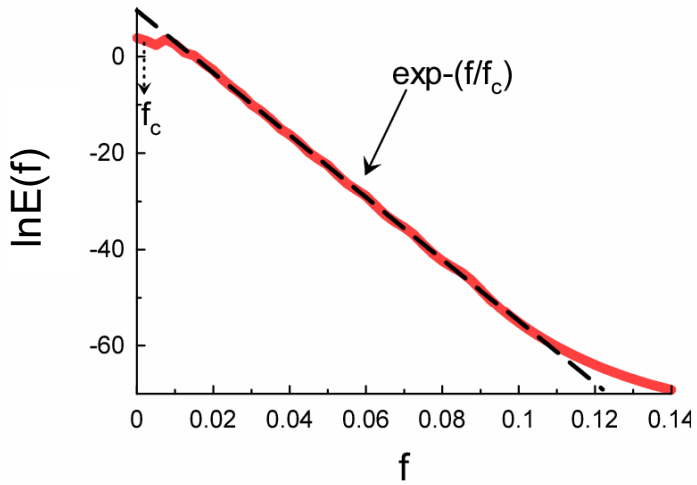

FIG. 4: Power spectrum of the $X(t)$ for the Lorenz system.

a Galerkin approximation of the Eqs. (1-3) [16]

$$
\frac{d x}{d t}=\sigma(y-x), \quad \frac{d y}{d t}=r x-y-x z, \quad \frac{d z}{d t}=x y-b z
$$

In this system the variables $x(t), y(t)$ and $z(t)$ are proportional to the effective rate of convection and to the horizontal and vertical temperature variations respectively [17].

For certain values of the parameters $\sigma, r$, and $b$ the deterministic chaotic dynamics was discovered in this system [16], 17]. As we will see later on in this paper the Lorenz system can be still rather inspiring.

Figure 2 shows a typical example of Lorenz chaotic (strange) attractor. The trajectory passes around the two fixed point of attraction of the focus type and chaotically switches from one to another forming the two wings of the attractor. Figure 3 shows a short sample of the $X(t)$ in the chaotic regime. The invariance of the Eq. (11) under the (reflective) coordinate transformation

$$
(x, y, z) \longrightarrow(-x,-y, z)
$$

(called rotation symmetry around the z-axis) will be useful for understanding of the helical nature of the abrupt chaotic inversions (reversals) of the large-scale circulation (wind) in the real thermal convection (see next Section).

The Lorenz system is a bounded and smooth dynamical system. Deterministic chaos in such systems is usually associated with the exponential frequency spectrum [18]-21]

$$
E(f) \propto \exp \left(-f / f_{c}\right)
$$

Figure 4, for instance, shows in the semi-log scales power spectrum for the $X(t)$-component of the Lorenz system. The dashed straight line indicates the exponential spectrum Eq. (13) and the short dotted arrow 


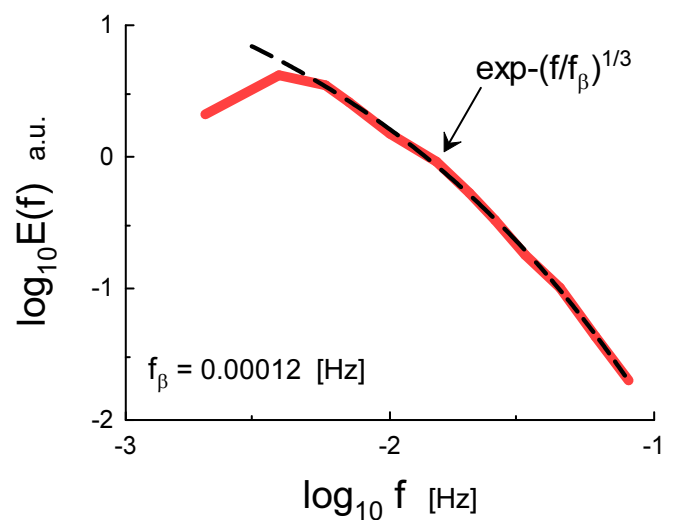

FIG. 5: Power spectrum of the local vertical velocity at $R a=$ $1.2 \cdot 10^{8}$.

indicates position of the $f_{c}$.

In the spatial domain the frequency spectrum Eq. (13) corresponds to the wavenumber spectrum

$$
E(k) \propto \exp \left(-k / k_{c}\right)
$$

(see Ref. [22] and references therein).

Increase of the number of the modes under consideration to a more realistic value results in fluctuations of the parameter $f_{c}$ in Eq. (13) and parameter $k_{c}$ in the Eq. (14).

An ensemble averaging over the fluctuating exponential spectrum

$$
E(k) \propto \int_{0}^{\infty} P\left(k_{c}\right) \exp -\left(k / k_{c}\right) d k_{c}
$$

can account this phenomenon and the stretched exponential spectrum

$$
E(k) \propto \exp -\left(k / k_{\beta}\right)^{\beta}
$$

can be considered as a generalization of the simple exponential one.

Comparing the Eqs. (15) and (16) we obtain an estimation of the large $k_{c}$ asymptotic of the probability distribution $P\left(k_{c}\right)$ in the Eq. (15) [23]

$$
P\left(k_{c}\right) \propto k_{c}^{-1+\beta /[2(1-\beta)]} \exp \left(-\gamma k_{c}^{\beta /(1-\beta)}\right)
$$

where $\gamma$ is a constant.

On the other hand, the probability distribution $P\left(k_{c}\right)$ can be obtained using simple dimensional considerations. Indeed, the scaling behaviour of the characteristic velocity $v_{c}$ for helicity dominated process can be estimated from the dimensional considerations as

$$
v_{c} \propto I^{1 / 4} k_{c}^{1 / 4} \text {. }
$$

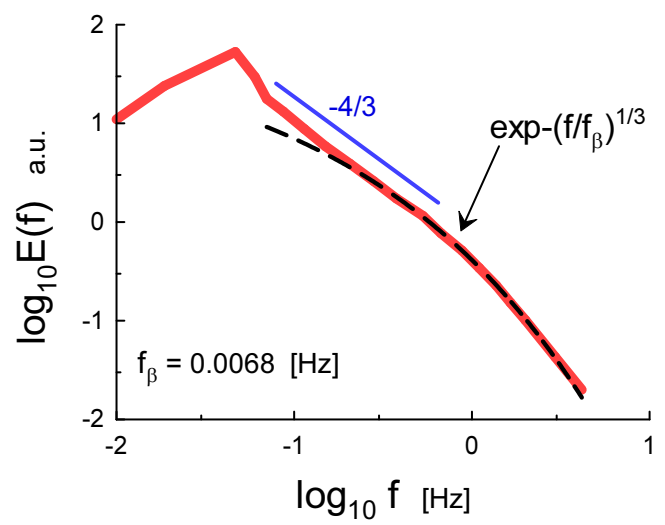

FIG. 6: Power spectrum of the local vertical velocity at $R a=$ $2.3 \cdot 10^{10}$

It directly follows from the Eq. (18) that if $v_{c}$ has a Gaussian distribution [12], then

$$
P\left(k_{c}\right) \propto k_{c}^{-3 / 4} \exp -b k^{1 / 2}
$$

Comparing Eqs. (17) and (19) and taking $\gamma=b$ one obtains $\beta=1 / 3$, i.e.

$$
E(k) \propto \exp -\left(k / k_{\beta}\right)^{1 / 3} .
$$

where $k_{\beta}$ is a renormalized (due to the fluctuations) characteristic wavenumber.

In Ref. [24] results of the laser Doppler velocimetry measurements of Rayleigh-Bénard convection in a cylindrical cell (filled with water) were reported. Local vertical velocity near the vertical sidewall of the cell was measured at mid-height of the cell at different values of the Rayleigh number (from $R a=1.2 \cdot 10^{8}$ to $R a=2.3 \cdot 10^{10}$ ). A large-scale circulation was also present in this experiment and the measurements were made within the rotational plane of the circulation. The aspect ratio of the cell $\Gamma=D / H$ (where $D$ is the cell's diameter) was close to 1.

Figures 5 and 6 show power spectra of the local vertical velocity at $R a=1.2 \cdot 10^{8}$ and $R a=2.3 \cdot 10^{10}$, respectively (the spectral data were taken from Fig. 2 of the Ref. 24]). The dashed curve indicates correspondence to the spectral law Eq. (20) (again the Taylor hypothesis has been applied). The straight line with the slope "-4/3" (cf. Eq. (10)) has been drawn in the Fig. 6 for reference.

\section{LARGE-SCALE CIRCULATION}

Figure 7 schematically shows how the cold (blue) and hot (red) plumes generate the large-scale circulation in the turbulent Rayleigh-Bénard convection (adapted from Ref. 25]). This schematic picture was confirmed 


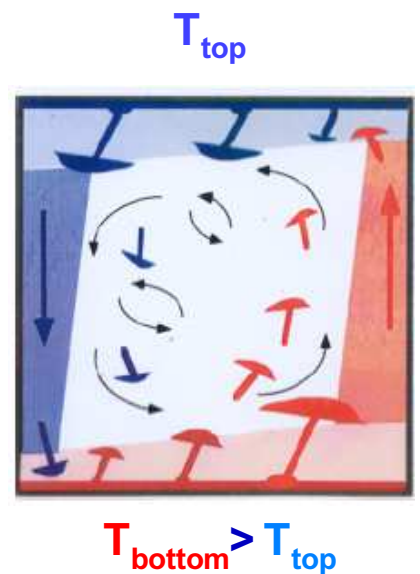

FIG. 7: Kadanoff's cartoon of generation of the large-scale circulation in the turbulent Rayleigh-Bénard convection.

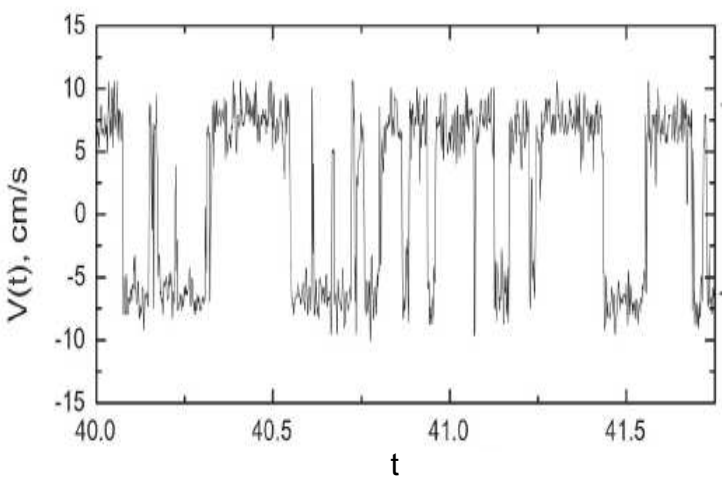

FIG. 8: A short sample of the time series of the large-scale circulation velocity at $R a=1.5 \cdot 10^{11}$.

by direct observations in the laboratory experiments (see, for instance, Ref. [26] and references therein).

Figure 8 (adapted from the Ref. [6]) shows a short sample of the time series of the large-scale circulation velocity measured in a cylindrical cell with $\Gamma=1$ (filled with cryogenic helium gas) near the vertical sidewall at mid-height of the cell at rather large value of the Rayleigh number $R a=1.5 \cdot 10^{11}$. Figure 9 shows power spectrum of the large-scale circulation velocity (the spectral data were taken from Fig. 2 of the Ref. [6]). The straight line is drawn in the Fig. 9 to indicate correspondence to the spectral law Eq. (10).

It should be noted that the large-scale circulation was also well observed at considerably smaller values of the Rayleigh number $R a=5.6 \cdot 10^{9}$ in a water-filled cylindrical Rayleigh-Bénard convection cell $(\Gamma=1)[26]$, for

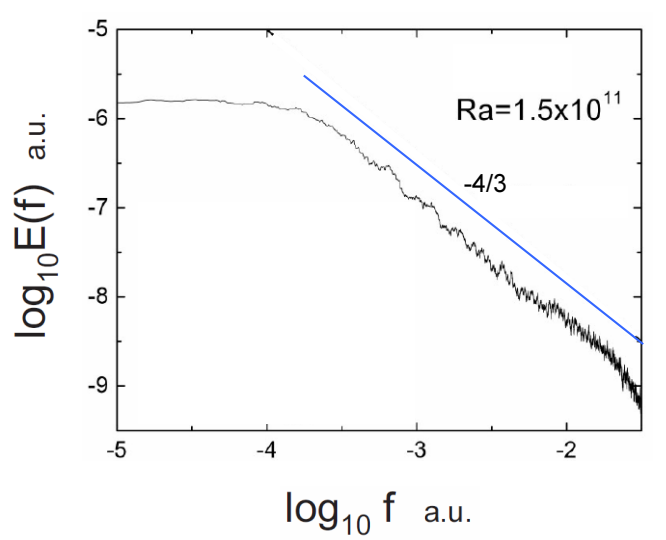

FIG. 9: Power spectrum of the large-scale circulation velocity at $R a=1.5 \cdot 10^{11}$.

instance.

Figures 10 and 11 show the large-scale circulation velocity spectra obtained in this experiment at the top and at the bottom of the cell correspondingly. Figure 12 shows the large-scale circulation velocity spectrum obtained in this experiment at the top of the cell at $R a=5.7 \cdot 10^{10}$ and $\Gamma=1 / 2$ (at the bottom of the cell the spectrum is analogous). The spectral data were taken from Fig. 7 of the Ref. [26].

The dashed curves in the Figs. 10-12 indicate correspondence to the spectral law Eq. (20). One can see that in this case (unlike the previous one - Fig. 9) the spectral behaviour indicates the distributed chaos instead of the scaling. In the both experiments, however, the large scale circulation was dominated by the second order moment of helicity distribution (the Levich-Tsinober invariant) - Eqs. (10) and (18-20).

The appearance of the clockwise (or alternatively the anticlockwise) large-scale circulation in the cylindrical cell is, of course, a spontaneous breaking of the reflectional symmetry. The observed phenomenon of the chaotic reversals of direction of the large-scale circulation (see Fig. 8, for instance) can be considered as an ergodic restoration of the global reflectional symmetry in a long run (cf. also Fig. 3 and its relation to the reflectional symmetry). Therefore, we can expect that (as in the case of the Lorenz attractor, see Section IV) we have deal with a two-wing attractor and chaotic transitions between the wings resulting in the wind reversals. Then, naturally, the each wing of the attractor can be associated with the mean helicity of the opposite sign (let us recall that the mean helicity is not conserved in the Rayleigh-Bénard convection) and the chaotic reversals of the large-scale circulation can be associated with reversals of the mean helicity's sign at the chaotic transitions from one wing of the attractor to another (cf. Ref. 27] and references therein). 


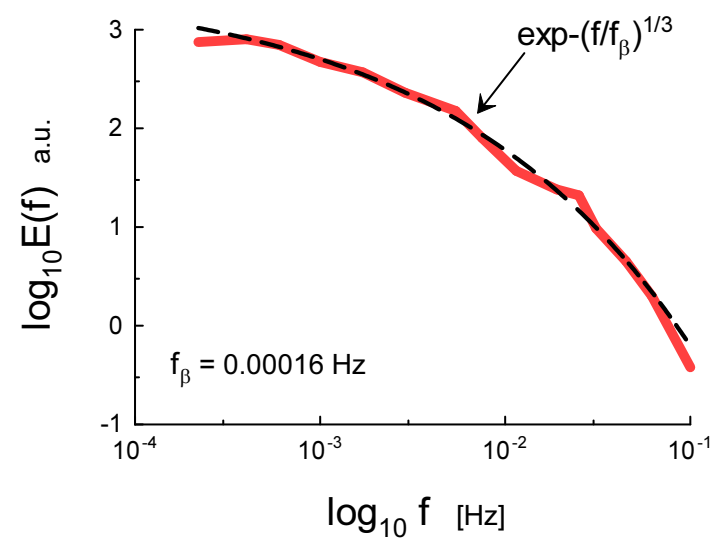

FIG. 10: Power spectrum of the large-scale circulation velocity at $R a=5.6 \cdot 10^{9}$ (at the top of the cell).

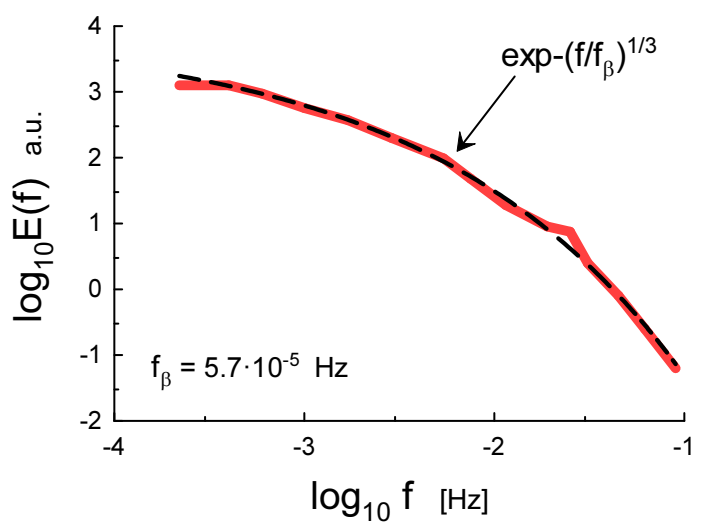

FIG. 11: Power spectrum of the large-scale circulation velocity at $R a=5.6 \cdot 10^{9}$ (at the bottom of the cell).

\section{GEOMAGNETIC DYNAMO}

In the Boussinesq approximation the dynamo action induced by thermal convection in an unstably stratified shell between two concentric spheres rotating with angular velocity $\Omega$ is described by the system of nondimensional equations

$$
\begin{gathered}
E \cdot\left\{\frac{\partial \mathbf{u}}{\partial t}+(\mathbf{u} \cdot \nabla) \mathbf{u}-\nabla^{2} \mathbf{u}\right\}+\nabla p=\mathbf{F}(\mathbf{u}, \mathbf{B}, T) \\
\mathbf{F}(\mathbf{u}, \mathbf{B}, T)=2[\hat{\mathbf{z}} \times \mathbf{u}]+R a \frac{\mathbf{r}}{R} T+P m^{-1}[(\nabla \times \mathbf{B}) \times \mathbf{B}] \\
\frac{\partial T}{\partial t}+(\mathbf{u} \cdot \nabla) T=P r^{-1} \nabla^{2} T
\end{gathered}
$$

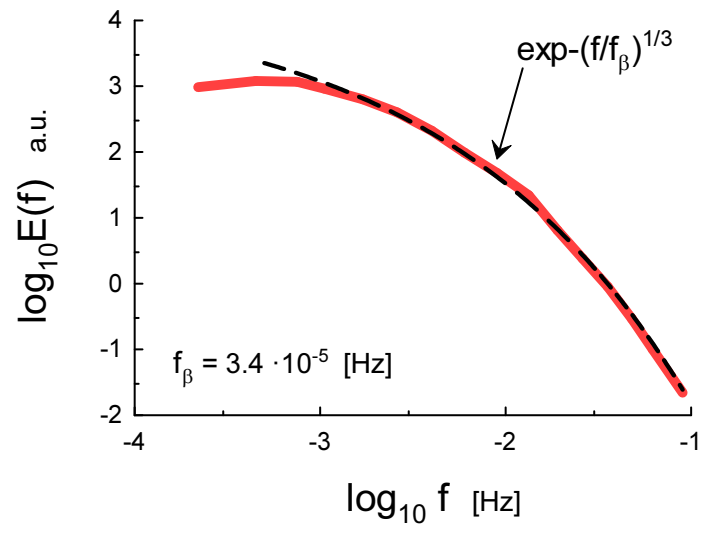

FIG. 12: Power spectrum of the large-scale circulation velocity at $R a=5.7 \cdot 10^{10}$ (at the top of the cell).

$$
\begin{gathered}
\frac{\partial \mathbf{B}}{\partial t}=\nabla \times(\mathbf{u} \times \mathbf{B})+P m^{-1} \nabla^{2} \mathbf{B} \\
\nabla \cdot \mathbf{u}=0, \quad \nabla \cdot \mathbf{B}=0
\end{gathered}
$$

with appropriate boundary conditions (see, for instance, Ref. [3] and references therein). In these equations $T$ is temperature, $\mathbf{B}$ is magnetic field, $\hat{\mathbf{z}}$ is a unit vector in the direction of the axis of rotation, $\mathbf{r}$ is the position vector and $R$ is the outer radius of the shell. Dimensionless $E=\nu / \Omega H^{2}$ is the Ekman number ( $H$ is the shell gap), $\operatorname{Pr}=\nu / \kappa$ is the Prandtl number, $\operatorname{Pm}=\nu / \eta$ is the magnetic Prandtl number ( $\eta$ is the magnetic diffusivity), $R a=\sigma g \Delta T H / \nu \Omega$ is the modified Rayleigh number $(\Delta T$ is the temperature difference between outer and inner boundaries of the shell).

For inviscid fluid $(\nu=0)$ the equation for mean helicity is

$$
\frac{d\langle h\rangle}{d t}=2\langle\boldsymbol{\omega} \cdot \mathbf{F}(\mathbf{u}, \mathbf{B}, T)\rangle
$$

(cf. the Eq. (5)). Hence the helicity is not an inviscid invariant in this case as well. However, the second order moment of the helicity distribution $I$ at certain conditions can be an adiabatic invariant of the system Eqs. (21-25). Indeed, if the main contribution to the generally non-zero correlation $\langle\boldsymbol{\omega} \cdot \mathbf{F}(\mathbf{u}, \mathbf{B}, T)\rangle$ is provided by the large-scale (mainly coherent) fields, but the correlation $\langle\boldsymbol{\omega} \cdot \mathbf{F}(\mathbf{u}, \mathbf{B}, T)\rangle$ is quickly weakened toward smaller spatial scales (due to the randomization produced by the turbulent effects), then the consideration similar to that of the Section II supports the adiabatic invariance of the second order moment of the helicity distribution 
I. Therefore, the two types of the spatial (wavenumber) spectrum related to this adiabatic invariance - Eqs. (10) and (20), can be also obtained for the velocity field generated by the system Eqs. (21-25) with the appropriate boundary conditions.

The equations (21-25) are dimensionless, but since the spectra Eqs. (10) and (20) have been obtained from the dimensional considerations let us recall that in the Alfvénic units the magnetic field has the same dimension as velocity 28]. Therefore, in these units the same dimensional considerations that were used in order to obtain the velocity spectrum Eq. (10) or Eq. (20) can be also used to obtain spectrum of the magnetic field in the same form of the Eq. (10) or (20).

Figure 13 shows the time- and volume-averaged kinetic and magnetic energy spectra computed in a recent fine resolved numerical simulation with $E=3 \times 10^{-7}$ and magnetic Reynolds number $R e_{m}=274$ in a spherical shell imitating the outer (metallic liquid) core of the Earth (the spectral data were taken from Fig. $3 \mathrm{~b}$ of the Ref. [29]). In this simulation the diffusivities of magnetic field and momentum were significantly different from one another $(P m=\nu / \eta=0.05)$. Therefore, most of the energy is dissipated Ohmically rather than viscously (similarly to the dissipation processes in the Earth's outer core). Also a uniform heat source was included in the Eq. (23).

The power spectra are shown against spherical harmonic degree $l$. The wave number $k$ corresponding to the spherical harmonic degree $l$ on a sphere of radius $r$ is [30]

$$
k=\frac{\sqrt{l(l+1)}}{r}
$$

Therefore, for sufficiently large $l$ the wavenumber $k \simeq$ $l / r$. The solid straight line is drawn in the Fig. 13 to indicate the spectral law Eq. (10) for the kinetic energy spectrum, whereas the dashed (lime) curve indicates the spectrum Eq. (20) (cf. next Section - Fig. 14, and Ref. [31]).

\section{CRUSTAL MAGNETIC FIELDS}

The directly measured geomagnetic field is a superposition of the main field, presumably generated by the geodynamo in the outer (liquid) core and the magnetic field of magnetized rocks in the crust. The main field dominates the comparatively long wavelengths, whereas the crustal geomagnetic field originating from magnetized crustal rocks dominates the geomagnetic power spectrum at the wavelengths between 0.1 and $100 \mathrm{~km}$ 32 .

The crustal magnetic filed is composed from induced and remanent magnetisation. The remanent (paleo-) magnetism, is a permanent magnetism in rocks,

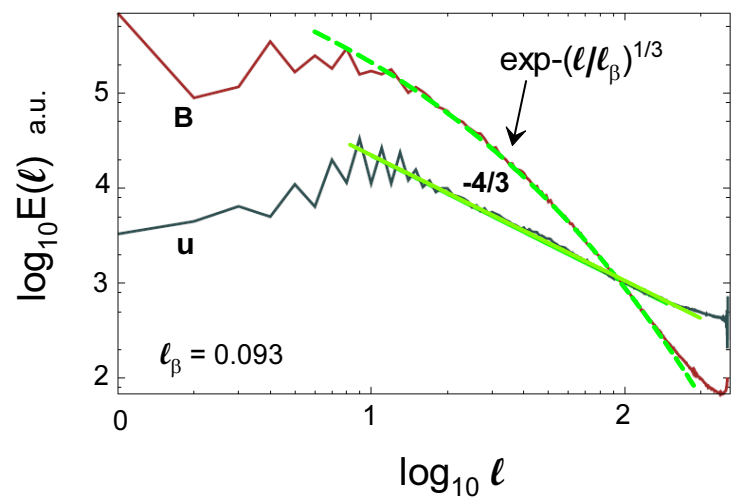

FIG. 13: Time- and volume-averaged kinetic and magnetic energy spectra.

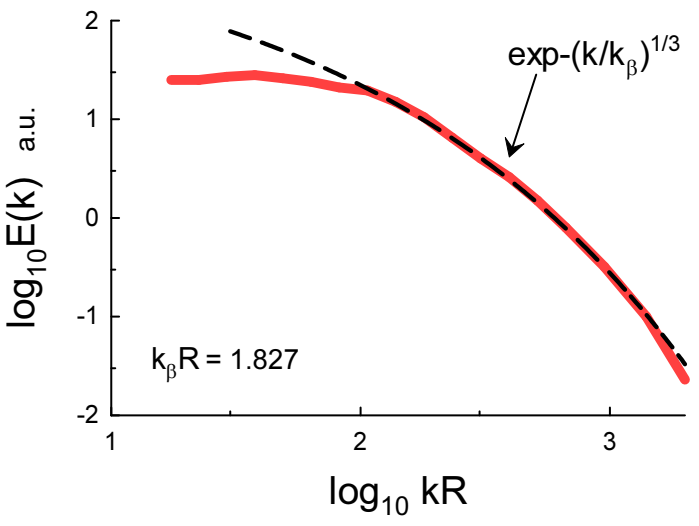

FIG. 14: Crustal magnetic power spectrum computed using the World Magnetic Anomaly Map.

originating at the time of the rocks formation. The remanent magnetisation dominates the comparatively short wavelength and it is, naturally, strong near the Earth surface.

On the other hand, the short-wavelength part of the crustal geomagnetic field is closely correlating with the near-surface geologic variations (it is especially strong in the vicinity of magnetic and ferrous geological materials). Therefore, it is not clear whether the remanent (paleo-) magnetism in the Earth crust will be globally dominating factor at the short wavelengths. If we assume a random distribution of the geologic variations on a global map, then this hypothesis can be readily verified.

Figure 13 shows geomagnetic power spectrum computed using subgrids $\left(20^{\circ} \times 20^{\circ}\right)$ of the National Geophysics Data Center's (NGDC's) World Magnetic Anomaly Map (WMAM [9]). The mean field was sub- 
tracted, and the data were detrended and azimuthally averaged (the spectral data were taken from Fig. 4 of the Ref. [33]).

The dashed curve in the Fig. 13 indicates correspondence to the spectral law Eq. (20). The $k_{\beta} R \simeq 1.827$ ( $R=6,371 \mathrm{~km}$ is the mean Earth's radius). Hence $1 / k_{\beta} \simeq 3,487 \mathrm{~km}$. Thus the characteristic spatial scale $1 / k_{\beta}$ is approximately equal to the radius of the outer

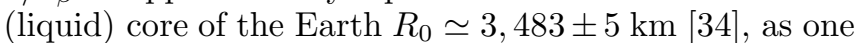
can expect for the geomagnetic dynamo.

\section{THE GEOMAGNETIC FIELD DIPOLE VARIABILITY}

In this Section we will be interested in the true temporal (frequency) spectra of the velocity field. The scaling temporal spectrum corresponding to the spatial one Eq. (10) can be readily obtained from the dimensional considerations:

$$
E(f) \propto \varepsilon_{I}^{4 / 5} f^{-7 / 5}
$$

As for the distributed chaos one should replace the spatial (wavenumber) Eq. (18) by corresponding temporal (frequency) relationship

$$
v_{c} \propto I^{1 / 5} f_{c}^{1 / 5} .
$$

Then, in the way similar to that used in the Section IV one obtains for the distributed chaos the stretched exponential temporal (frequency) spectrum

$$
E(f) \propto \exp -\left(f / f_{\beta}\right)^{2 / 7} \text {. }
$$

where $f_{\beta}$ is a renormalized (due to the fluctuations) characteristic frequency (cf. the Eq. (20)).

The equations (21-25) are dimensionless, but since the spectra Eqs. (27) and (29) have been obtained from the dimensional considerations let us recall that in the Alfvénic units [28] the magnetic field has the same dimension as velocity. Therefore, in these units the same dimensional considerations that were used in order to obtain the velocity spectrum Eq. (27) or Eq. (29) can be also used to obtain spectrum of the magnetic field in the same form of the Eq. (27) or (29).

The paleo-geomagnetic data is usually obtained for the geomagnetic dipole moment. The magnetic dipole moment normalized by the domain's volume $V$ is

$$
\boldsymbol{\mu}=\frac{1}{2 V} \int[\mathbf{r} \times \mathbf{j}] d V=\frac{1}{2 V} \int[\mathbf{r} \times(\nabla \times \mathbf{B})] d V
$$

and in the Alfvénic units the $\boldsymbol{\mu}$ also has the same dimension as velocity, and the same dimensional considerations can be used in this case as well. Therefore the scaling Eq. (27) and stretched exponential Eq. (29) spectra can

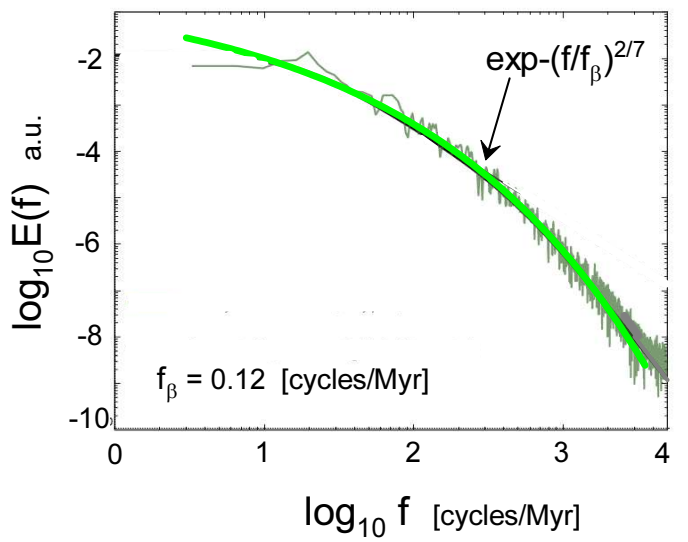

FIG. 15: Power spectrum of the axial dipole magnetic field (numerical simulation, $R m=90$ ).

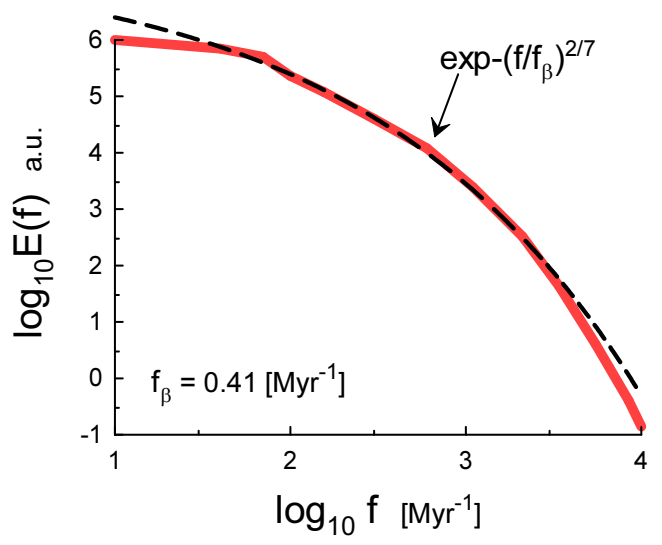

FIG. 16: Power spectrum of the axial dipole magnetic field (numerical simulation, $R m=564$ ).

be used for the geomagnetic dipole moment dynamics.

Figure 14 shows power spectrum of the axial dipole magnetic field obtained in a thermal convection-driven geodynamo simulation reported in Refs. [35], 36] (the spectral data were taken from Fig. 2 of the Ref 36] for the magnetic Reynolds number $R m=90)$. The system Eqs. (21-25) were used at this simulation with appropriate boundary conditions at $E=5 \cdot 10^{5}$, $R a=1400, P m=0.5$ and $P r=1$. The lime curve is drawn to indicate the spectrum Eq. (29).

Figure 15 shows power spectrum of the axial dipole magnetic field obtained in another thermal convectiondriven geodynamo simulation reported in Ref. [37] (the spectral data were taken from Fig. 3 of the Ref [37] for the magnetic Reynolds number $R m=564$ ). The system Eqs. (21-25) were used at this simulation with 


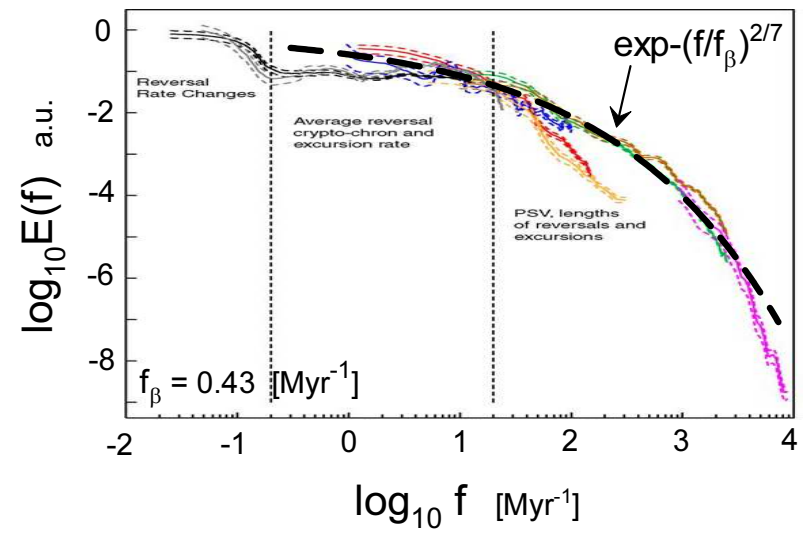

FIG. 17: Composite power spectrum of geomagnetic dipole moment variations for the time interval 0-160 Myr.

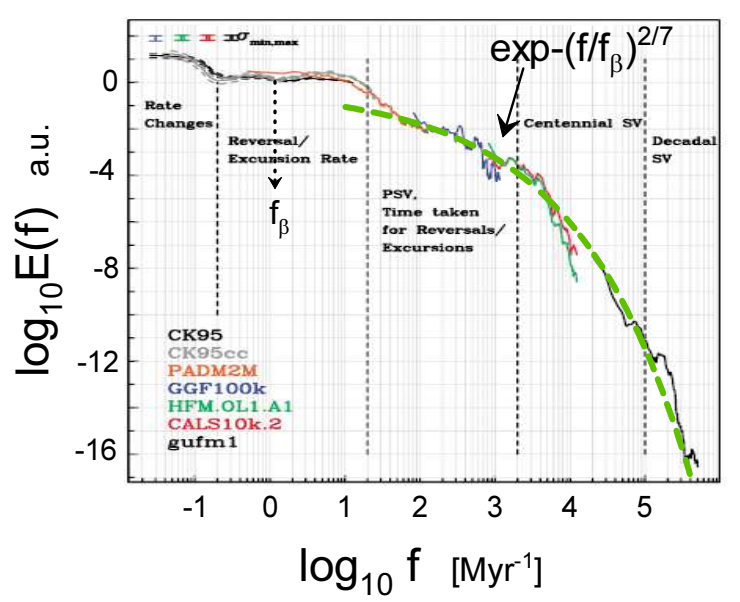

FIG. 18: Extended composite (with a modified composition) power spectrum of geomagnetic dipole moment variations for the time interval 0-160 Myr.

appropriate boundary conditions and some additional constraints (see for details Ref. [37]). The dashed curve is drawn to indicate the spectrum Eq. (29) (the distributed chaos).

Figure 16 shows a composite power spectrum of the geomagnetic field dipole variability and reversals for the time interval 0-160 Myr (the spectrum was adapted from the Fig. 7 of the Ref. [38]). The magnetostratigraphic time scale, different marine sediment paleointensity records and a paleomagnetic field model were used to construct the composite spectrum. The thick dashed curve indicates correspondence to the spectrum Eq. (29).

Figure 17 shows power spectrum of the geomagnetic dipole moment composed from CK95 reversal record (0-160 Myr), CK95cc reversal record cryptochrons (0$83 \mathrm{Myr})$, PADM2M axial dipole moment reconstruction (0-2 Myr), HFM.OL1.A1 and CALS10k.2 Holocene geomagnetic field models, gufm1 historical geomagnetic field model, and GGF100k geomagnetic field model (0100kyr). The thick dashed curve is drawn to indicate correspondence to the spectrum Eq. (29), and the dotted arrow indicates position of the $f_{\beta}$ (the spectral data have been adapted from Fig. 8 of the Ref. [40]).

\section{CONCLUSIONS}

From the consideration presented in this paper one can conclude that the thermal convection-driven geomagnetic dipole variability and the crustal remanent magnetization are apparently dominated by the second order moment of the helicity distribution in an inertial range of scales inside the Earth's outer (liquid) core.

Comparing this result with the above discussed abrupt chaotic reversals of the large-scale circulation in the pure thermal convection one can expect that the observed abrupt chaotic reversals of the geomagnetic field polarity are related to the ergodic reversals in mean helicity sign as well, and the observed dynamics of the geomagnetic field can provide qualitative and quantitative information about dynamics of velocity field in the Earth's outer (liquid) core.
[1] J.E.T. Channell and L. Vigliotti, Reviews of Geophysics, 57, 709 (2019)

[2] K. Glassmeier and J. Vogt, Space Sci. Rev., 155, 387 (2010).

[3] H. Amit, R. Leonhardt and J. Wicht, Space Sci. Rev., 155, 293 (2010)

[4] E. Levich and A. Tsinober, Phys. Lett. A 93, 293 (1983)

[5] H.K. Moffatt and A. Tsinober, Annu. Rev. Fluid Mech., 24, 281 (1992)

[6] J.J. Niemela, L. Skrbek, K.R. Sreenivasan and R.J. Don- nelly, J. Low Temp. Phys., 126, 297 (2002)

[7] K.R. Sreenivasan, A Bershadskii, J.J. Niemela, Phys. Rev. E, 056306 (2002)

[8] A. Bershadskii, Chaos, 20, 043124 (2010)

[9] S. Maus, T. Sazonova, et. al., K. Hemant, Geochem. Geophys. Geosystems, 8, Q06017 (2007)

[10] A. Kumar, A.G. Chatterjee and M.K. Verma, Phys. Rev. E, 90, 023016 (2014)

[11] A. Bershadskii and A. Tsinober, Phys. Rev. E, 48, 282 (1993). 
[12] A. S. Monin, A. M. Yaglom, Statistical Fluid Mechanics, Vol. II: Mechanics of Turbulence (Dover Pub. NY, 2007)

[13] A.A. Grachev, Boundary-Layer Meteorology, 69, 27 (1994)

[14] H. Tennekes, J. Fluid Mech., 67, 561 (1975)

[15] A. Kumar and M.K. Verma, R. Soc. open sci., 5, 172152 (2018)

[16] E.N. Lorenz, J. Atmos. Sci., 20, 130 (1963)

[17] C. Sparrow, The Lorenz Equations: Bifurcations, Chaos, and Strange Attractors (Springer-Verlag, 1982)

[18] N. Ohtomo, K. Tokiwano, Y. Tanaka et. al., J. Phys. Soc. Jpn., 64, 1104 (1995)

[19] D.E. Sigeti, Phys. Rev. E, 52, 2443 (1995)

[20] J. D. Farmer, Physica D, 4, 366 (1982).

[21] U. Frisch and R. Morf, Phys. Rev., 23, 2673 (1981)

[22] J. E. Maggs and G. J. Morales, Phys. Rev. Lett., 107, 185003 (2011); Phys. Rev. E 86, 015401(R) (2012); Plasma Phys. Control. Fusion, 54, 124041 (2012)

[23] D.C. Johnston, Phys. Rev. B, 74, 184430 (2006)

[24] X-D. Shang and K-Q. Xia, Phys. Rev E, 64, 065301(R0 (2001)

[25] L.P. Kadanoff, Annu. Rev. Cond. Matt. Phys., 6, 1 (2015)

[26] H.-D. Xi and K.-Q. Xia, Phys. Rev. E, 78, 036326 (2008)

[27] E. Levich, Concept. Phys., VI(3), 239 (2009)

[28] R. Moll, J. Pietarila Graham, J. Pratt, R. H. Cameron, W.-C. Muller, and M. Schussler, Astrophys. J., 736, 36
(2011)

[29] A. Sheyko, C. Finlay, J. Favre and A. Jackson, Sci. Rep., 8, 12566 (2018)

[30] G. Backus, R.L. Parker and C. Constable, Foundations of Geomagnetism (Cambridge Univ. Press, Cambridge UK, 1996)

[31] A. Bershadskii, Res. Notes AAS, 4, 10 (2020)

[32] V. Lesur, D. Gubbins, Geophys. J. Intern., 142, 889 (2000)

[33] S. Maus, Geophys. J. Int., 174, 135 (2008)

[34] T.J. Ahrens, ed., Global earth physics a handbook of physical constants (3rd ed., Washington, AGU, 1995) Geophysical Union.

[35] B.A. Buffett, E.M. King and H. Matsui, Geophys. J. Int., 198, 597 (2014)

[36] B.A. Buffett and H. Matsui, Earth and Planet. Sci. Lett., 411, 20 (2015)

[37] J. Davies and C.G. Constable, Earth and Planet. Sci. Lett., 404, 238 (2014)

[38] C. Constable and C. Johnson, Phys. Earth and Planet. Interiors, 153, 61 (2005)

[39] J-P. Valet, L. Meynadier and Y. Guyodo, Nature, 435, $802(2005)$

[40] S. Panovska, C.G. Constable and M.Korte, Geochem. Geophys. Geosystems, 19, 4757 (2018) 